\title{
Binding energy of static perfect fluids.
}

\author{
Janusz Karkowski and Edward Malec \\ Institute of Physics, Jagiellonian University, 30-059 Cracow, Reymonta 4, Poland.
}

\begin{abstract}
We investigate the binding energy in two classes of polytropic perfect fluids. A general-relativistic bound from below is derived in the case of a static compact body, having the same form as in the newtonian limit. It is shown that the positivity of the binding energy implies that the (properly defined) average speed of sound is smaller than the escape velocity. A necessary condition for the negative binding energy, stating that the maximal speed of sound is close to the escape velocity, is found in a class of fluids.
\end{abstract}

\section{INTRODUCTION}

There are two popular classes of equations of states of perfect fluids. One, that is commonly used by astrophysicists, takes a simple form when formulated in terms of the so-called baryonic number density. The other class, preferred by general relativists, uses the mass density. The two classes coincide in the newtonian limit but they can lead to markedly different predictions in the strong field regime (see, for instance, a comparative discussion of stationary accretion in [1]). It is intention of this paper to rederive some known results and obtain new ones on the binding energy of perfect fluids satisfying polytropic equations of state of the two types. A possible new feature of the derivation is that we do not recourse to the laws of relativistic thermodynamics. We restrict our attention to static fluid bodies, but since we operate in those classes of equations of state for which staticity implies spherical symmetry [2], we end in investigating spherically symmetric objects.

The order of this paper is as follows. Section 2 presents equations of selfgravitating fluids. In the third section we recall definitions of the two classes of relativistic equations of state and show that in the (properly defined) Newtonian limit they coincide. Section 4 defines the gravitational binding energy and in Secs 5 its value is estimated in the polytropic subclass of the equations of state. In the newtonian limit the binding energy agrees with the standard newtonian expression. Section 6 is dedicated to the derivation of certain inequalities onto the gravitational potential energy. It is remarkable that these inequalities are exact, and one of them has the same form both in the general-relativistic and in the newtonian cases. Section 7 presents the main results. It is shown that the binding energy of a compact spherical body is bounded from below by the standard newtonian formula $-P /(\Gamma-1)+3 m_{T B}^{2} /(5 R)$. The kinetic energy of the gas is represented by the total pressure $P$ and the sufficient condition for having a positive value of the binding en- ergy is that $P$ is smaller than the gravitational potential type term $3 m_{T B}^{2} /(5 R)$. That can be translated into a bound from above onto a quantity that represents a kind of an average speed of sound - that should not exceed the escape velocity (or even the first cosmic velocity). A necessary condition (a lower bound on the maximal speed of sound) for the negative binding energy is formulated in the so-called $\tilde{p}-\rho$ class of fluids.

\section{EQUATIONS FOR SELFGRAVITATING FLUIDS.}

Throughout this paper the Newtonian constant $G$ and the velocity of light $c$ are put equal to 1 . Let the selfgravitating system be spherically symmetric. Then the whole dynamics is carried by a material field characterized by an energy-momentum tensor $T_{\mu \nu}$. In the case of the perfect fluid $T_{\mu \nu}=(\tilde{p}+\rho) U_{\mu} U_{\nu}+\tilde{p} g_{\mu \nu}$, where the coordinate velocity is timelike, $U_{\mu} U^{\mu}=-1$.

Assuming spherical symmetry, one can deal with the metric

$$
d s^{2}=-N^{2} d t^{2}+\alpha d r^{2}+R^{2} d \Omega^{2}
$$

where $N, R$ and $\alpha$ are unknown metric functions. In sections 2, 3 and in the first part of Sec. 4 we work in comoving coordinates. Our aim there is to justify a particular expression of the binding energy, and that requires some information about the evolution that led to the formation of a bound system. Comoving coordinates are convenient in describing evolving fluids. Starting from section 4 we use standard (polar gauge) coordinates.

The needed material quantities are the energy density $\rho=-T_{0}^{0}$ and the isotropic pressure, $\tilde{p}=T_{r}^{r}=T_{\theta}^{\theta}=T_{\phi}^{\phi}$. Define $p R \equiv 2 \partial_{r} R / \sqrt{\alpha}$ (this is so-called mean curvature of a centered sphere [4]) and $U \equiv \partial_{t} R / N$. One can show, using the constraint equations [3] and the properties of comoving coordinates, that

$$
p R=2 \sqrt{1-2 \frac{m(R)}{R}+U^{2}},
$$

where $m(R)$ is the quasilocal mass specified below. The trace of the extrinsic curvature

$$
\operatorname{tr} K=K_{i}^{i} \equiv \frac{1}{N} \partial_{0} \ln \sqrt{\operatorname{det}\left(g_{i j}^{3}\right)}
$$

can be found from the momentum constraint [3],

$$
\operatorname{tr} K=2 \frac{\partial_{r}\left(U R^{2}\right)}{\sqrt{a} R^{3} p} .
$$


The continuity equations $\nabla_{i} T_{j}^{i}=0$ reduce to two equations

$$
\begin{gathered}
N \partial_{r} \tilde{p}+\partial_{r} N(\tilde{p}+\rho)=0, \\
\partial_{0} \rho=-N \operatorname{tr} K(\tilde{p}+\rho) .
\end{gathered}
$$

The Einstein evolution equations reduce to the single equation

$$
\partial_{0} U=-N \frac{m}{R^{2}}+\frac{\partial_{r} R \partial_{r} N}{\alpha}-4 \pi N R \tilde{p} .
$$

The quasilocal mass ("mass function")

$$
m(r)=\int_{V(r)} d V \frac{R p}{2} \rho
$$

contained within a coordinate sphere $r^{\prime} \leq r$ satisfies the evolution law

$$
\partial_{0} m(r)=-4 \pi N R^{2} U \tilde{p} .
$$

The mass $m(r)$ (which happens to be equal to the volume representation of the Hawking mass [4]) can be expressed also as

$$
m(R(r))=4 \pi \int_{0}^{R(r)} d r r^{2} \rho ;
$$

the integrand is now written in terms of the areal radius. The total mass is conserved; the asymptotic mass $m(\infty)$ is constant on the whole foliation that develops from a given initial hypersurface. There exists another mass measure, known as the rest mass [5],

$$
M(R)=\int_{V(R)} d V \rho=8 \pi \int_{0}^{R} d r \frac{r}{p} \rho .
$$

The rest mass $M(\infty)$ is not conserved - it might change from a slice to a slice (that is with time). In what follows we will write $M$ and $m$ instead of $M(\infty)$ and $m(\infty)$

The baryonic mass (the mass density $n$ is defined in the next section) contained within a coordinate sphere $r$,

$$
m_{b a r}(r)=\int_{V(r)} d V n=8 \pi \int_{0}^{R(r)} d r \frac{r}{p} n,
$$

is conserved: $\partial_{0} m_{\text {bar }}(r)=0$. That follows easily from equations (16), (6) and (3). Yet another mass characteristic of a static body comprised within the coordinate sphere $r$ is its Trautman-Bondi mass $m_{T B}$, equal to the quasilocal mass function $m(r)$. This mass is equal to the asymptotic mass minus that fraction of the fluid that has been dispersed away to infinity. Therefore $m_{T B} \leq m$.

\section{EQUATIONS OF STATE}

The ( $\tilde{p}-n$ henceforth) equation of state used by astrophysicists is

$$
\tilde{p}=\tilde{p}(n)
$$

while the community of general relativists prefers $(\tilde{p}-\rho$ thereafter)

$$
\tilde{p}=\tilde{p}(\rho) .
$$

Here $n$ is the baryonic mass density that can be defined (up to a normalization factor) as an integrability factor that ensures the conservation of the baryonic current

$$
j^{\mu}=n U^{\mu} .
$$

One can easily show [3] that the conservation laws $\nabla_{\mu} T_{\nu}^{\mu}=0$ imply, under conditions assumed above, the relation

$$
n=n_{0} \exp \left(\int_{\rho_{0}}^{\rho} d s \frac{1}{s+\tilde{p}(s)}\right) .
$$

Here $n_{0}$ and $\rho_{0}$ are constants that can be used in order to suitably normalize $n$. Thus, given $\rho$ and an equation of state $\tilde{p}=\tilde{p}(\rho)$ satisfying $\nabla_{\mu} T_{\nu}^{\mu}=0$, one finds $n$ automatically satisfying the continuity equation $\nabla_{\mu}\left(n U^{\mu}\right)=0$.

And conversely, given conserved baryonic density and an equation of state $\tilde{p}=\tilde{p}(n)$ one can find $\rho$,

$$
\rho=n \int_{0}^{n} d s \frac{\tilde{p}(s)}{s^{2}}+n,
$$

such that $\nabla_{\mu} T_{\nu}^{\mu}=0$. The formulae are particularly simple in the case of polytropic equations of state. Assuming

$$
\tilde{p}=K \rho^{\Gamma}
$$

with $\Gamma>1$ one has

$$
n=n_{0} \frac{\rho}{\left(1+\frac{a^{2}}{\Gamma}\right)^{\frac{1}{\Gamma-1}}}
$$

( $a$ is the speed of sound). If one assumes $n_{0}=1$ then, defining the newtonian limit to be the case $a^{2} \ll 1$, it follows that in the newtonian limit the baryonic density and the mass density are very close, $n \approx \rho$.

Assuming the alternative state equation,

$$
\tilde{p}=K n^{\Gamma},
$$

one obtains from Eq. (17)

$$
\rho=n+\frac{\tilde{p}}{\Gamma-1} ;
$$

if the newtonian limit is understood as the stronger than before condition $\tilde{p} /(\Gamma-1) \ll 1$, then $\partial_{\rho} n \approx 1$ and again 
$n \approx \rho$. Assuming in Eq. (19) that $n_{0} \rightarrow 0$ and simultaneously keeping the pressure $\tilde{p}$ fixed (that is $K n_{0}^{\Gamma}=$ const), one obtains the Harrison et al. equation of state [6]

$$
\rho=\frac{\tilde{p}}{\Gamma-1} .
$$

In the following part of this work we shall deal with equations of state given by (18) and (20). The adiabatic index $\Gamma$ is assumed to be strictly bigger than 1 . While the above relations are well known, it is interesting that the above derivation can be done solely within classical general relativity, without appealing to thermodynamics.

\section{BINDING ENERGY}

The binding energy $E$ of a static system $S$ can be defined as the difference between the initial mass that is needeed in order to create $S$ and the final TrautmanBondi mass. The most natural definition would be to put $E=m-m_{T B} ; E$ is the difference between the asymptotic (ADM) mass $m$ and the mass $m_{T B}$ of the final product. Traditionally one defines the binding energy somewhat differently, using the concept of the baryonic mass. We will adhere to the standard approach, defining another measure $E_{b i n d}$ of the binding energy; the reader may check later that $E \geq E_{\text {bind }}$.

In the case of initial mass distributions that are at rest and so dispersed that the curvature of the Cauchy hypersurface is practically negligible, the two masses $m$ and $M$ can be practically equal. If we assume that matter consists exclusively of baryons, then (again, under the condition that the fluid is so diluted that its pressure is negligible) the baryonic mass $m_{b a r}(r)$ and the mass function $m(r)$ can be regarded as being equal initially. Since usually only a fraction of the gas (say, comprised within the coordinate sphere $r$ ) becomes solidified in a form of the static object $S$, and it is the baryonic mass $m_{\text {bar }}(r)$ that remains constant during a collapse, it is justifiable to define the binding energy $E$ as [7]

$$
E_{b i n d}=m_{b a r}(r)-m_{T B} .
$$

Let us point out that the baryonic mass can be expressed - using formulae of Sec. 3 - in terms of quantities describing the static system $S$. From now on we will employ standard (Landau-Lifschitz [8] ) coordinates with the radial coordinate being always the areal radius. This implies $\alpha=1 /\left(1-\frac{2 m(R)}{R}\right)$, where the mass function is given by Eq. (10). All volume integrals will be performed over the volume $V$ of $S$. In order to simplify notation, the total rest mass $M(r)$ of $S$ will be denoted by $M_{S}$ and the binding baryonic mass $m_{\text {bar }}(r)$ will be written simply as $m_{\text {bar }}$. Notice that $m_{T B}$ is this quantity that enters the newtonian formula describing the late-time interaction of $S$ with a distant (but located at a finite distance) test body.

Let us define total pressure in $S$ by

$$
P=\int_{V} d V \tilde{p}=8 \pi \int_{0}^{R} d r \frac{r}{p} \tilde{p} .
$$

It is convenient to rewrite formula (23) in two different forms,

$$
E_{b i n d}=M_{S}-\frac{P}{\Gamma-1}-m_{T B} .
$$

in the case of the $\tilde{p}-n$ equation of state and

$$
E_{b i n d}=\int_{V} d V \frac{\rho}{\left(1+\frac{a^{2}}{\Gamma}\right)^{\frac{1}{\Gamma-1}}}-m_{T B} .
$$

$\tilde{p}-\rho$ equation of state. The equivalence of (26) and (25) with (23) follows from (21) and (19), respectively.

In the newtonian limit both expressions yield the same quantity

$$
E_{b i n d}=\int_{V} d V\left(\frac{\rho m(r)}{r}-\frac{\tilde{p}}{\Gamma-1}\right) .
$$

\section{ESTIMATES OF THE BINDING ENERGY}

One can invoke to a workable version of the virial theorem, that exists in static spherically symmetric systems, in order to eliminate $P$ from $(25)$ ( [9] - [11]). This is obtained from the Oppenheimer-Volkov equation

$$
(\tilde{p}+\rho)\left(\frac{m(R)}{R^{2}}+4 \pi \tilde{p} R\right)=-\partial_{R} \tilde{p}\left(1-\frac{2 m(R)}{R}\right) ;
$$

Multiplying (28) by $4 \pi R^{3} / \sqrt{1-\frac{2 m(r)}{r}}$ and integrating with respect $R$, one gets (integrating by parts, in order to eliminate the grad-term $\left.\partial_{R} \tilde{p}\right)$

$$
\begin{aligned}
& 3 \tilde{P} \equiv 3 \int_{V} d V \tilde{p}\left(1-\frac{2 m(R)}{R}\right)= \\
& -U_{G}+4 \pi \int_{V} d V \tilde{p} r^{2}(\tilde{p}+2 \rho) .
\end{aligned}
$$

Here $U_{G}$ is the gravitational self-interaction energy,

$$
U_{G} \equiv-\int_{V} d V \frac{\rho m(r)}{r}
$$

The total pressure $P=\int_{V} d V \tilde{p}$ is not smaller than $\tilde{P}$, as one can see rewriting the first line of (29)

$$
P=\tilde{P}+\int_{V} d V \frac{2 m(r) \tilde{p}}{r} .
$$

The mass difference $M_{S}-m_{T B}$ reads (this follows directly from definitions of the two masses) 


$$
M_{S}-m_{T B}=-\tilde{U}_{G}=\int_{V} d V \frac{2 \rho m(r)}{r\left(1+\sqrt{1-\frac{2 m(r)}{r}}\right)} .
$$

Define

$$
-\hat{U}_{G}=-\tilde{U}_{G}+\frac{1}{3 \Gamma-4} \int_{V} d V \frac{2 \rho m(r)^{2}}{r^{2}\left(1+\sqrt{1-\frac{2 m(r)}{r}}\right)^{2}} .
$$

Employing the above information, after some calculations one arrives at

$$
\begin{aligned}
& M_{S}-\frac{P}{\Gamma-1}-m_{T B}= \\
& M_{S}-\frac{\tilde{P}}{\Gamma-1}-m_{T B}-\frac{1}{\Gamma-1} \int_{V} d V \frac{2 m(r) \tilde{p}}{r}= \\
& -\frac{3 \Gamma-4}{3(\Gamma-1)} \hat{U}_{G}-\frac{1}{(\Gamma-1)} \int_{V} d V \frac{2 m(r) \tilde{p}}{r}- \\
& \frac{4 \pi}{3(\Gamma-1)} \int_{V} d V \tilde{p} r^{2}(\tilde{p}+2 \rho) .
\end{aligned}
$$

Thence,

$$
M_{S}-\frac{P}{\Gamma-1}-m_{T B} \leq-\frac{3 \Gamma-4}{3(\Gamma-1)} \hat{U}_{G} .
$$

Notice, that $E_{b i n d}=M_{S}-\frac{P}{\Gamma-1}-m_{T B}$ for the $\tilde{p}-n$ equation of state. Therefore we infer that the binding energy may become negative for values of the polytropic index $\Gamma \leq 4 / 3$. In the newtonian limit one obtains

$$
E_{b i n d}=-\frac{3 \Gamma-4}{3(\Gamma-1)} U_{G} .
$$

In the newtonian limit the binding energy becomes strictly negative only when $\Gamma<4 / 3$, while in the regime of strong gravitational fields this may happen even for $\Gamma=\epsilon+4 / 3$, for small but positive $\epsilon$. The general relativistic effects may decrease the binding energy (in agreement with [7]). Let us remark here, that the result in [7] corresponding to Eq. (36) has a different coefficient (for reasons not quite clear to us): $(3 \Gamma-4) /(5 \Gamma-6)$ instead of $(3 \Gamma-4) /(3(\Gamma-1))$.

Notice that always $E_{\text {bind }}<M_{S}-m_{T B}$. In the nonrelativistic case and putting $\Gamma=5 / 3$ one obtains a stronger result, $E_{b i n d}=-U_{G} / 2 \approx\left(M-m_{T B}\right) / 2$; the quantity $M_{S}-m_{B}$, that can be regarded as the gravitational potential energy in the case of static systems (or perhaps even momentarily static systems), bounds the binding energy and it is of the order of the binding energy. The second part of the last statement is not valid for strongly curved geometries, even in the case $\Gamma=5 / 3$.

Assuming the polytropic $\tilde{p}-\rho$ equation of state, we can express the binding energy by formula (26). It is easy to show that $\left(\frac{1}{1+\frac{a^{2}}{\Gamma}}\right)^{\frac{1}{\Gamma-1}} \geq 1-\frac{a^{2}}{\Gamma(\Gamma-1)}$. Therefore one arrives at

$$
E_{b i n d} \geq M_{S}-\frac{P}{\Gamma-1}-m_{T B} .
$$

It is clear that now the use of the virial theorem (which bounds from above $M_{S}-\frac{P}{\Gamma-1}-m_{T B}$ ) cannot give an estimate from above on the binding energy. There exists only a trivial bound $E_{\text {bind }} \leq-\tilde{U}_{G}$ (this is because $\left.M_{S} \geq m_{\text {bar }}\right)$ - the binding energy is bounded by the absolute value of the gravitational potential energy. One can see, comparing formulae (34) and (37), that the $\tilde{p}-\rho$ equation of state is more favourable for having nonnegative binding energy that the $\tilde{p}-n$ case. Nevertheless, in the newtonian limit one again recovers estimate (36). We will show in the last section that by exploiting the quantity $M-m_{T B}$ one can find another useful bound from below.

\section{BOUNDING POTENTIAL ENERGY}

In this section one might partly relax former conditions, and to deal with a compact (not necessarily fluid) body on the momentarily stationary spatial 3dimensional geometry. The assumptions i) of staticity, and ii) that matter is a barotropic fluid, $\tilde{p}=K \rho^{\Gamma(\rho)}$, become relevant in a refined version of the forthcoming result. To be specific, the second and third terms on the right hand side of Eq. (39) would be absent without the two last conditions. Let us point that the compactness of the fluid body imposes a bound from below on the barotropic index ( [12] and [13]).

It is easy to show the following result, that estimates the difference $M_{S}-m_{T B}$.

Theorem. Assume that matter has compact support enclosed within a sphere $R$, that no minimal surface does exist on the Cauchy slice and $\rho \geq 0$. Then

$$
\begin{aligned}
-\tilde{U}_{G}= & M_{S}-m_{T B} \geq \frac{m_{T B}^{2}}{R} \frac{2}{\left(1+\sqrt{1-\frac{2 m_{T B}}{R}}\right)^{2}} \geq \\
& \frac{m_{T B}^{2}}{2 R} .
\end{aligned}
$$

If in addition the configuration is static, then

$$
\begin{aligned}
-\tilde{U}_{G}= & M_{S}-m_{T B} \geq \frac{m_{T B}^{2}}{R} \frac{2}{\left(1+\sqrt{1-\frac{2 m_{T B}}{R}}\right)^{2}}- \\
& \frac{m_{T B}}{2\left(1+\sqrt{1-\frac{2 m_{T B}}{R}}\right)}+ \\
& \frac{3 R}{4}\left(-1+\sqrt{\frac{R}{2 m_{T B}}} \arcsin \left(\sqrt{\frac{2 m_{T B}}{R}}\right)\right) \geq \\
& \frac{3 m_{T B}^{2}}{5 R} .
\end{aligned}
$$


Proof of the theorem. Straightforward calculation allows one to check that

$$
\begin{aligned}
& M_{S}=\frac{1}{2} \int_{0}^{R} d r\left(-2 \sqrt{r} \frac{d}{d r} \sqrt{r-2 m(r)}+\frac{1}{\sqrt{1-\frac{2 m(r)}{r}}}\right)= \\
& -R \sqrt{1-\frac{2 m_{T B}}{R}}+\frac{1}{2} \int_{0}^{R} d r\left(\frac{1}{\sqrt{1-\frac{2 m}{r}}}+\sqrt{1-\frac{2 m(r)}{r}}\right) .
\end{aligned}
$$

The integrand of the last integral is bounded from below by 2 . Therefore $M_{S} \geq R\left(1-\sqrt{1-\frac{2 m_{T B}}{R}}\right)$; thus

$$
\begin{aligned}
& M_{S}-m_{T B} \geq R\left(1-\sqrt{1-\frac{2 m_{T B}}{R}}\right)-m_{T B}= \\
& \frac{m_{T B}^{2}}{R} \frac{2}{\left(1+\sqrt{1-\frac{2 m_{T B}}{R}}\right)^{2}} \geq \\
& \frac{m_{T B}^{2}}{2 R} .
\end{aligned}
$$

We can get only thus far in the case of momentarily stationary data.

If our configuration is static, then the OppenheimerVolkov equation implies $\partial_{R} \rho=a^{-2} \partial_{R} \tilde{p} \leq 0$. This in turn yields $\partial_{r}\left(m(r) / r^{3}\right) \leq 0$ (that result was originally derived by Buchdahl [14]).

Returning to Eq. (40), notice that

$$
\begin{aligned}
& \frac{1}{2} \int_{0}^{R} d r\left(\frac{1}{\sqrt{1-\frac{2 m}{r}}}+\sqrt{1-\frac{2 m(r)}{r}}\right)= \\
& R+\int_{0}^{R} \frac{d r}{r^{2}} \frac{2 m^{2}}{\sqrt{1-\frac{2 m}{r}}\left(1+\sqrt{1-\frac{2 m}{r}}\right)^{2}} .
\end{aligned}
$$

Since $m(r) / r^{3}$ is decreasing, we have $m(r) / r^{3} \geq$ $m_{T B} / R^{3}$. Therefore

$2 \int_{0}^{R} \frac{d r}{r^{2}} \frac{m^{2}}{\sqrt{1-\frac{2 m}{r}}\left(1+\sqrt{1-\frac{2 m}{r}}\right)^{2}} \geq$
$\frac{2 m_{T B}^{2}}{R^{6}} \int_{0}^{R} d r \frac{r^{4}}{\sqrt{1-\frac{2 m_{T B} r^{2}}{R^{3}}}\left(1+\sqrt{1-\frac{2 m_{T B} r^{2}}{R^{3}}}\right)^{2}}$

The second integral term in Eq. (43) can be explicitly evaluated, with the result

$$
\begin{aligned}
F \equiv & \frac{-m_{T B}}{2\left(1+\sqrt{1-\frac{2 m_{T B}}{R}}\right)}+ \\
& \frac{3 R}{4}\left(-1+\sqrt{\frac{R}{2 m_{T B}}} \arcsin \sqrt{\frac{2 m_{T B}}{R}}\right) .
\end{aligned}
$$

Combining (40), (43) and (44), one gets the first of the sought inequalities of (39). The second inequality there follows from the trivial estimate $4 \int_{0}^{R} d r \frac{r^{4}}{\sqrt{1-\frac{2 m_{T B} r^{2}}{R^{3}}}\left(1+\sqrt{1-\frac{2 m_{T B} r^{2}}{R^{3}}}\right)^{2}} \geq R^{5} / 5$. That accomplishes the proof of the theorem.

Let us again stress here that while in (38) one needs only that initial data are momentarily static, the result (4Q)ith the coefficient $3 / 5$ in (39) requires staticity (without that we would have only the coefficient $1 / 2$ ). The two results are exact. The first inequality is saturated by the spherical shell of matter (see an example studied in [15]) while the inequalities of (39) are saturated both in the general-relativistic and the newtonian cases, by a constant density star. In the case of fluid bodies satisfying the Buchdahl limit $R=9 m_{T B} / 4$ [14] one obtains $M_{S} \geq(-9 / 16+81 \arcsin (2 \sqrt{2} / 3) /(32 \sqrt{2})) m_{T B} \approx$ $1.65 m_{T B}$; that again becomes an equality for constant density stars. Constant density stars are the most economic configurations in the sense that they minimize the ratio $M_{S} / m_{T B}$ - and, consequently, the gravitational potential energy $-\tilde{U}$ - amongst the subset of static solutions.

\section{CONCLUSIONS}

Before pursuing further, one should be aware that the assumption of compactness of a fluid body made in Sec. 6 imposes some restrictions on the polytropic index. In the case of $\tilde{p}-\rho$ equations of state the condition is roughly $\Gamma>6 / 5$, but for the $\tilde{p}-n$ case the situation is unclear the application of existing results concerning the general barotropic models ( [9], [12] and [13]) requires a separate investigation. We assume that the polytropic index is just right to agree with the compactness.

Collecting together the results of Secs 5 and 6 , we arrive at the inequality, valid for both $\tilde{p}-n$ and $\tilde{p}-\rho$ models,

$$
\begin{aligned}
E_{\text {bind }} \geq & M_{S}-\frac{P}{\Gamma-1}-m_{T B} \geq \\
& -\frac{P}{\Gamma-1}+F+\frac{m_{T B}^{2}}{R} \frac{2}{\left(1+\sqrt{1-\frac{2 m_{T B}}{R}}\right)^{2}} .
\end{aligned}
$$

A weaker but more elegant than (45) is the inequality

$$
E_{\text {bind }} \geq-\frac{P}{\Gamma-1}+\frac{3 m_{T B}^{2}}{5 R},
$$

which retains the same form in the newtonian limit. The sufficient condition that the binding energy be positive is

$$
\frac{P}{\Gamma-1}<F+\frac{m_{T B}^{2}}{R} \frac{2}{\left(1+\sqrt{1-\frac{2 m_{T B}}{R}}\right)^{2}}
$$

or, more simply,

$$
\frac{3 m_{T B}^{2}}{5 R}>\frac{P}{\Gamma-1},
$$


both for $\tilde{p}-n$ and $\tilde{p}-\rho$ equations of state. An alternative form of a sufficient condition,

$$
\frac{P}{\Gamma-1}<\frac{M_{S}^{2}}{2 L}
$$

can be obtained due to another inequality $M_{S}-m_{T B} \geq$ $M_{S}^{2} /(2 L)$ (where $L$ is the geodesic radius of a compact body), proven in [15]. There exists a simple interpretation of this condition in terms of the speed of sound. Namely, define an "average speed of sound $\tilde{a}$ " by $P \equiv \tilde{a}^{2} M_{S} / \Gamma$. The quantity $\tilde{a}$ is bounded from below and above by the minimal $a_{\min }$ and maximal $a_{\max }$ speeds of sound, respectively, within the compact body. The formerly formulated sufficient condition implies now

$$
\tilde{a}^{2}<\Gamma(\Gamma-1) \frac{3 m_{T B}^{2}}{5 R M_{S}} .
$$

But $M_{S} \geq m_{T B}$; therefore

$$
\tilde{a}^{2}<\Gamma(\Gamma-1) \frac{3 m_{T B}}{5 R}
$$

as the consequence of the positivity of the binding energy. In the newtonian limit $M_{S} \approx m_{T B}$ and (49) becomes just another sufficiency condition for $E_{b i n d}>0$. This speed of sound should not be bigger than (roughly) the escape velocity (or, strictly saying, the first cosmic velocity $\left.m_{T B} / R\right)$, if $\Gamma \leq 5 / 3$, and it decreases with the decrease of $\Gamma$. And conversely, the inequality $(\tilde{a})^{2} \geq \Gamma(\Gamma-1) \frac{3 m_{T B}^{2}}{5 R M_{S}}$ is necessary for $M_{S}-\frac{P}{\Gamma-1}-m_{T B}$ being negative, and thus necessary for the binding energy being negative.

Another form of the necessary condition can be found for $\tilde{p}-\rho$ equations of state. It follows from (26) that

$$
E_{b i n d}=\int_{V} d V n-m_{T B} \geq \frac{M_{S}}{\left(1+\frac{a_{\max }^{2}}{\Gamma}\right)^{\frac{1}{\Gamma-1}}}-m_{T B} .
$$

The use of the estimate (39) of the Theorem allows us to say that $E_{b i n d}$ can be negative only if

$$
\begin{aligned}
& a_{\max }^{2} \geq \Gamma\left(1+F+\frac{m_{T B}}{R} \frac{2}{\left(1+\sqrt{1-\frac{2 m_{T B}}{R}}\right)^{2}}\right)^{\Gamma-1} \\
& -\Gamma \text {, }
\end{aligned}
$$

or (in a simpler and weaker form)

$$
a_{\max }^{2} \geq \Gamma\left[\left(1+\frac{3 m_{T B}}{5 R}\right)^{\Gamma-1}-1\right] .
$$

Since $x \equiv \frac{3 m_{T B}}{5 R} \leq 4 / 15$, one has $(1+x)^{\delta} \geq 1+\delta\left(\frac{15}{19}\right)^{\delta-1}$. Therefore Eq. (52) can be written as

$$
a_{\max }^{2} \geq \frac{3}{5} \Gamma(\Gamma-1)\left(\frac{15}{19}\right)^{2-\Gamma} \frac{m_{T B}}{R} .
$$

This clearly demonstrates, that the negative binding energy requires the maximal speed of sound to be close to the escape velocity. For compact objects satisfying the Buchdahl limit we get from (51), putting $\Gamma=5 / 3$, the necessary condition $a_{\max }^{2}>0.66$. Let us remark that (52) and (53) yield much worse estimates $a_{\max }^{2}>0.28$ and $a_{\max }^{2}>0.27$, respectively. In such a case the upper limit for the maximal allowed kinetic energy (with $\left.E_{b i n d}>0\right)$ exceeds $(\Gamma-1) \times 0.65 m_{T B}$ (see the end of the preceding section); the kinetic energy can constitute a significant fraction of the mass $m_{T B}$.

By reversing the argument, one obtains from the converse of (51) - (53) a set of sufficiency conditions for the positivity of $E_{b i n d}$. The strongest statement is that if the maximal speed of sound is smaller than the right hand side of (51), then $E_{\text {bind }}>0$.

As is well known, the negative sign of the binding energy is correlated with the instability of static fluids [7]. Thus inequalities (48) and (51 - 53) agree well with the intuitive notion that hot systems (i. e., with high speed of sound) are unstable. It is also remarkable that the upper limit increases monotonically with the increase of $\Gamma$.

We conjecture that inequalities (45 - 53) hold true for systems with the general barotropic equation $\tilde{p}=K \rho^{\Gamma(\rho)}$ (under a suitable definition of the constants). A plausible form of a related condition in the case of stationary nonspherical fluid bodies can be $\frac{P}{\Gamma-1}<m_{T B}^{2} / \sqrt{\frac{S}{\pi}}$.

Acknowledgments. This work has been supported in part by the KBN grant 2 PO3B 00623 .

[1] B. Kinasiewicz and T. Lanczewski, Comparison of two stationary spherical accretion models, gr-qc/0404019.

[2] R. Beig and W. Simon, Commun. Math. Phys. 144, 373(1992); R. Beig and W. Simon, Lett. Math. Phys. 21, 245(1991).

[3] E. Malec, Phys. Rev. D60, (1999)104043.

[4] E. Malec and N. O' Murchadha, Phys. Rev. D50, 6036(1994).

[5] R. Wald, General Relativity, (Chicago, University of Chicago Press, 1984).

[6] B. K. Harrison, K. S. Thorne, M. Wakano and J. A. Wheeler, Gravitation Theory and Gravitational Collapse, (Chicago, University of Chicago Press 1965) - a quote after [7].

[7] R. F. Tooper, Astrophysical J., 142, 1541(1965).

[8] L. Landau and L. Lifschitz, Classical Field Theory, vol. 2, Nauka, Moscow 1973 (in Russian).

[9] W. Simon, Springer Lecture Notes in Physics, vol. 604, 223(2002).

[10] S. Bonazzola, Astrophysics J. 182, 335(1973).

[11] A. Georgiou, Class. Quant. Grav., 20, 359(2003). 
[12] W. Simon, Class. Quant. Grav. 10, 177(1993).

[13] J. M. Heinzle, Class. Quant. Grav. 19, 2835(2002) and references therein.

[14] H. A. Buchdahl, Phys. Rev., 116, 1027(1957).

[15] E. Malec, P. Bizoń and N. O' Murchadha, Class. Quant. Grav. 7, 1953(1990); E. Malec and P. Koc, ibid, L199. 